\title{
Safwat Marzouk
}

\section{Egypt as a Monster in the Book of Ezekiel}

[Ägypten als Ungeheuer im Buch Ezechiel.]

Veröffentlicht auf Englisch.

Safwat Marzouk nimmt Bezug auf die Ungeheuer Theorie und das antike nahöstliche Motiv des »Chaoskampfs « und zeigt,

SAFWAT MARZOUK

Egypt as a Monster in the Book of Ezekiel

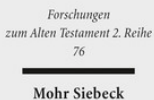

2015. XVI, 289 Seiten. FAT II 76

SBN 978-3-16-153628-1

eBook PDF 104,00

ISBN 978-3-16-153245-0

fadengeheftete Broschur $104,00 €$ dass der paradoxe Charakter der Kategorie des Untiers der Grund ist, warum Ägypten im Buch Ezechiel als Seeungeheuer dargestellt wird. Obwohl es von außen betrachtet so scheint, als würde das Ungeheuer schiere Andersartigkeit verkörpern, befindet sich darunter eine verstörende Ähnlichkeit. Obwohl das Monster besiegt und sein Körper verstümmelt, wird es nie vollkommen zerstört. Ägypten wird im Buch Ezechiel als Seeungeheuer dargestellt, weil Ägypten die Bedrohung durch religiöse Assimilation verkörpert. Obwohl diese Monstrosität Ägyptens ursprünglich wegen gemeinsamer Identitätselemente zwischen Ägypten und Israel konstruiert wurde, transformiert der Prophet diese Darstellung des Monsters, um Ägypten als monströses Anderes darzustellen. In einem Kampfmythos besiegt YHWH das Ungeheuer und verstümmelt seinen Körper.

Safwat Marzouk Born 1978; PhD in Biblical Studies at Princeton Theological Seminary; ordained Presbyterian pastor with the Synod of the Nile and assistant professor of OT/HB at the Anabaptist Mennonite Biblical Seminary, Elkhart Indiana.

Jetzt bestellen:

https://mohrsiebeck.com/buch/egypt-as-a-monster-in-the-book-of-ezekiel-9783161536281?no_cache=

order@mohrsiebeck.com

Telefon: $+49(0) 7071-923-17$

Telefax: +49 (0)7071-51104 